\title{
Constraint Choice and Model Selection in the Generalized Maximum Entropy Principle ${ }^{+}$
}

\author{
Andrea Somazzi ${ }^{1}$ and Diego Garlaschelli ${ }^{2}$ \\ 1 Scuola Normale Superiore di Pisa, Pisa, Italy \\ 2 IMT School for Advanced Studies Lucca, Lucca, Italy \\ + Presented at the Entropy 2021: The Scientific Tool of the 21st Century, 5-7 May 2021; Available online: \\ https://sciforum.net/conference/Entropy2021/.
}

Published: 5 May 2021

The maximum entropy principle (MEP) is a powerful statistical inference tool that provides a rigorous way to guess the probability distribution of the states of a system which is known only through partial information.

Generalizing the Shore and Johnson's axioms, Jos Uffink (1995) proved that the functionals which are suitable to be used in the MEP belong to a one-parameter family, which the Shannon entropy is a member of. The resulting probability distributions are generalized exponentials, of which Boltzmann distribution is a special case. It has been discussed (P. Jizba and J. Korbel, 2019) that this generalized approach is suitable to study systems which do not respect standard hypothesis such as ergodicity, short-range interactions or exponential growth of the sample space: the resulting probability distributions take into account correlations that may not have been observed.

In this presentation, the maximum likelihood method to evaluate the parameters of such distributions and to perform model selection starting from empirical data will be discussed.

In particular, it will be shown that the maximum likelihood approach to estimate the Lagrange multipliers leads to an equation that justifies the use of the q-generalized momenta as constraints in the entropy maximization. Moreover, it will be shown that the likelihood function spontaneously emerges from the maximization of entropy: in particular, it will be proved that the log-likelihood is equal to minus the entropy once the Lagrange multipliers are fixed to satisfy the maximum likelihood condition.

Lastly, simple examples based on synthetic data will be presented to show that this approach provides accurate estimations of the parameters.

(C) 2021 by the authors. Licensee MDPI, Basel, Switzerland. This article is an open access article distributed under the terms and conditions of the Creative Commons Attribution (CC BY) license (http://creativecommons.org/licenses/by/4.0/). 